\title{
Application of a fiber-reinforced continuum theory to multiple deformations of the annulus fibrosus
}

\author{
Stephen M. Klisch - University of California, San Francisco, and University of California, Berkeley \\ Jeffrey C. Lotz - University of California, Berkeley
}

\begin{abstract}
Accurate tissue stress predictions for the annulus fibrosus are essential for understanding the factors that cause or contribute to disc degeneration and mechanical failure. Current computational models used to predict in vivo disc stresses utilize material laws for annular tissue that are not rigorously validated against experimental data. Consequently, predictions of disc stress resulting from physical activities may be inaccurate and therefore unreliable as a basis for defining mechanical-biologic injury criteria. To address this need we present a model for the annulus as an isotropic ground substance reinforced with two families of collagen fibers, and an approach for determining the material constants by simultaneous consideration of multiple experimental data sets. Two strain energy functions for the annulus are proposed and used in the theory to derive the constitutive equations relating the stress to pure stretch deformations. These equations are applied to four distinct experimental protocols and the material constants are determined from a simultaneous, nonlinear regression analysis. Good agreement between theory and experiment is achieved when the invariants are included within multiple, separate exponentials in the strain energy function.
\end{abstract}

\section{Introduction}

Intervertebral disc degeneration is a common clinical finding and a primary etiologic factor for spinal instability and low back pain (Deyo et al., 1991; Vanharanta et al., 1988). The hallmarks of this condition are dehydration of the normally gelatinous nucleus pulposus and progressive disorganization of the annulus fibrosus. In health, the annulus is a composite tissue consisting of collagen fibers arranged in layered sheets (lamellae) with a structure similar to that of an angle-ply laminate (Marchand and Ahmed, 1990; Tsuji et al., 1993). This architecture is ideal for containing the pressurized nucleus pulposus that, in turn, supports spinal compressive loads. As disc degeneration progresses, the annulus loses its lamellar organization and develops clefts and proteoglycanous cysts within its midsubstance, leading to protrusion and potential compression of adjacent nerve roots and/or the spinal cord (Lipson, 1988; Yamada, 1970). The epidemiological association between excessive physical activity and disc degeneration suggests that improper tissue stress may potentiate injury. This can occur acutely by way of direct extracellular matrix disruption (Vernon-Roberts and Pirie, 1977; Yasuma, 1990), or chronically via cellular remodeling (Brickley-Parsons and Glimcher, 1984; Lotz et al., 1998). In either case, the pathomechanics of this process is not understood to the extent that it may serve as a basis for elective clinical interventions. Toward that goal, the foundation for improved understanding of disc degeneration requires the ability to precisely define the stress with the disc in vivo. Spatial and temporal correlations between tissue stress predictions and the biologic (and morphologic) changes observed in in vivo models of degeneration can be used to clarify the mechanisms of degeneration and the bounds of tissue injury tolerance.

Because of the inherent difficulties associated with direct measurement of annular stresses in situ, engineering techniques are typically used to simulate the response of disc to physiologic loading, thereby predicting stresses. 


\section{Nomenclature}

$\begin{array}{ll}\otimes & \text { dyadic tensor product } \\ \mathbf{F} & \text { deformation gradient tensor } \\ \mathbf{T} & \text { Cauchy stress tensor } \\ W & \text { strain energy function } \\ \mathbf{C} & \text { right Cauchy-Green deformation ten- } \\ & \text { sor } \\ I_{3} & \text { determinant of } \mathbf{C} \\ \mathbf{B} & \text { left Cauchy-Green deformation ten- } \\ & \text { sor } \\ J & \text { determinant of } \mathbf{F} \\ \mathbf{P} & \text { first non-symmetric Piola-Kirchhoff } \\ & \text { stress tensor }\end{array}$

$\left(\mathbf{a}_{0}, \mathbf{b}_{0}\right),(\mathbf{a}, \mathbf{b})$ fiber direction vectors in the reference and present configurations, respectively

$\left(\mathbf{E}_{1}, \mathbf{E}_{2}, \mathbf{E}_{3}\right) \quad$ rectangular Cartesian basis vectors in the reference configuration

$I_{\mathrm{i}}$,

$2 \Phi$

I

$\lambda_{i}$,

$n ; \beta_{i}$

$\alpha_{i}$

$T^{\prime}$

$\delta_{i j}$

$T_{i}$

$\chi^{2}$ ents are needed but cannot be determined explicitly. Previous attempts at using the continuum approach were limited by: (1) the assumption of incompressibility ( $\mathrm{Wu}$ and Yao, 1976) (annular tissue is now known to posses significant compressibility (Iatridis et al., 1998)); (2) assumption of linearity (Elliott et al., 1997) (the annulus demonstrates significant nonlinearity under physiologic loads (Shirazi-Adl et al., 1986)); and (3) the use of data from only a single type of experiment (Galante, 1967; Fujita et al., 1997; Ebara et al., 1996; Iatridis et al., 1998).

The objective of this study was to develop a nonlinear, orthotropic constitutive law for the solid phase of the annulus fibrosus that, in the future, could be used to provide accurate predictions of the in vivo stresses within disc tissue. Further, because of the morphological complexity of the annulus, we questioned whether sufficient information exists within individual experiments to adequately define the mechanical behavior. Alternatively, we sought to simultaneously consider data from multiple experimental deformations to prescribe the requisite material constants. In developing our constitutive model, we utilized a theory for an isotropic continuum reinforced with two families of fibers (Spencer, 1984).

\section{Methods}

\subsection{Fiber-reinforced continuum theory}

The constitutive equation for a Green elastic material takes the form

$\mathbf{T}=\frac{2}{\sqrt{I_{3}}} \mathbf{F} \frac{\partial W}{\partial C} \mathbf{F}^{\mathbf{T}}$,

where $\mathbf{T}$ is the Cauchy stress, $\mathbf{F}$ is the deformation gradient tensor, a superscript $\mathrm{T}$ denotes the matrix transpose operator, $I_{3}$ is the determinant of the right Cauchy-Green deformation tensor $\mathbf{C}=\mathbf{F}^{\mathrm{T}} \mathbf{F}, W=W(\mathbf{C})$ is the strain energy, and $\partial W / \partial \mathbf{C}=\frac{1}{2}(\partial W / \partial \mathbf{C}+$ $(\mathrm{d} W / \mathrm{d} C) T)$. Alternatively, using the first (nonsymmetric) Piola-Kirchhoff stress tensor $\mathbf{P}$ defined by $J \mathbf{T}=\mathbf{P F}^{\mathbf{T}}$ where $J$ is the determinant of $\mathbf{F}$, Eq. (1) can be written as

$\mathbf{P}=2 \mathbf{F} \frac{\partial W}{\partial \mathbf{C}}$.

In the theory for a fiber-reinforced continuum, it is assumed that the anisotropic properties of the material are solely attributed to the presence of the families of fibers (Spencer, 1984). For two families of fibers, the directions of the fiber elements are represented by material line elements in the reference configuration, denoted by the vectors $\mathbf{a}_{0}$ and $\mathbf{b}_{0}$. These material line elements are deformed into the vectors $\mathbf{a}$ and $\mathbf{b}$ in the present configuration. To model the annulus, we take the rectangular Cartesian basis vectors $\left(\mathbf{E}_{1}, \mathbf{E}_{2}, \mathbf{E}_{3}\right)$ to be aligned in the approach where the behavior of the individual constitu- 


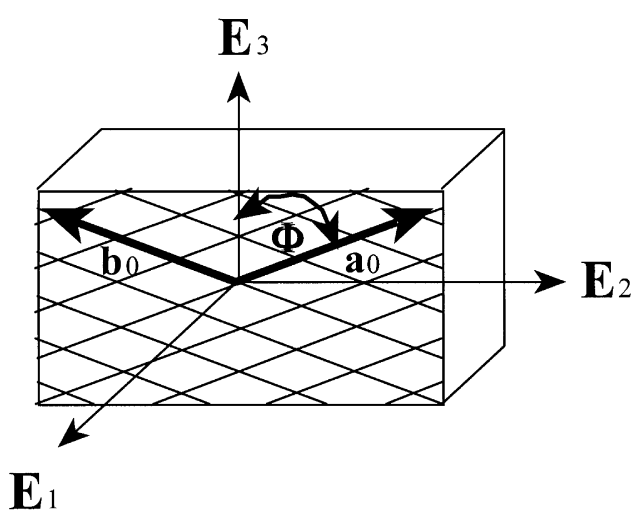

Fig. 1. Continuum idealization of the annulus fibrosus in the reference configuration. The basis vectors $\left(\mathbf{E}_{1}, \mathbf{E}_{2}, \mathbf{E}_{3}\right)$ are aligned in the local radial, circumferential, and axial directions, respectively. The two familites of fibers are represented in this reference configuration by the vectors $\mathbf{a}_{0}$ and $\mathbf{b}_{0}$ which lie in the $\mathbf{E}_{2}-\mathbf{E}_{3}$ plane and are oriented at angles of plus and minus $\Phi$ degrees from the axial direction $\mathbf{E}_{3}$.

radial, circumferential, and axial directions of a local anatomical configuration (Fig. 1). We define

$\mathbf{a}_{0}=\sin \Phi \mathbf{E}_{2}+\cos \Phi \mathbf{E}_{3}$,

$\mathbf{b}_{0}=-\sin \Phi \mathbf{E}_{2}+\cos \Phi \mathbf{E}_{3}$

so that the two families of fibers are oriented at angles of plus and minus $\Phi$ degrees from the axial direction. It is assumed that $W$ is an isotropic scalar function of the tensors $\left(\mathbf{C}, \mathbf{a}_{0} \otimes \mathbf{a}_{0}, \mathbf{b}_{0} \otimes \mathbf{b}_{0}\right)$, and that the two fiber families are mechanically equivalent such that $W$ is symmetric with respect to interchanging the fiber directions $\mathbf{a}_{0}$ and $\mathbf{b}_{0}$ (the dyadic product $\otimes$ is defined by $(\mathbf{a} \otimes \mathbf{b}) \mathbf{c}=\mathbf{a}(\mathbf{b} \bullet \mathbf{c})$ for all vectors $\mathbf{a}, \mathbf{b}$, and $\mathbf{c}$ and where $\cdot$ denotes the dot product of two vectors). With these assumptions, Spencer (1984) showed that $W$ can be expressed as

$W=\tilde{W}\left(I_{1}, I_{2}, I_{3}, I_{8}, I_{9}, I_{10}, I_{11}, \cos ^{2} 2 \Phi\right)$,

where

$I_{1}=\operatorname{tr} \mathbf{C}, \quad I_{2}=\frac{1}{2}\left[(\operatorname{tr} \mathbf{C})^{2}-\operatorname{tr} \mathbf{C}^{2}\right], \quad I_{3}=\operatorname{det} \mathbf{C}$,

$I_{4}=\mathbf{a}_{0} \cdot \mathbf{C} \mathbf{a}_{0}, \quad I_{5}=\mathbf{a}_{0} \cdot \mathbf{C}^{2} \mathbf{a}_{0}, \quad I_{6}=\mathbf{b}_{0} \cdot \mathbf{C} \mathbf{b}_{0}$,

$I_{7}=\mathbf{b}_{0} \cdot \mathbf{C}^{2} \mathbf{b}_{0}, \quad I_{8}=\cos 2 \Phi \mathbf{a}_{0} \cdot \mathbf{C} \mathbf{b}_{0}, \quad I_{9}=I_{4}+I_{6}$,

$I_{10}=I_{4} I_{6}, \quad I_{11}=I_{5}+I_{7}$

and $2 \Phi$ is the angle between the fiber families in the reference configuration. The invariants $I_{4}$ and $I_{6}$ are equal to the stretches along the fiber directions $\mathbf{a}_{0}$ and $\mathbf{b}_{0}$, respectively. Also, the invariants $I_{4}, I_{5}, I_{6}$, and $I_{7}$ only appear in the combinations shown in (5) due to the assumption that the fiber families are mechanically equivalent. Using (1) and (4) we can derive the constitutive equation for the Cauchy stress in terms of the left Cauchy-Green deformation tensor $\mathbf{B}=\mathbf{F F}^{\mathrm{T}}$ :

$$
\begin{aligned}
\mathbf{T}= & \frac{2}{I_{3}^{1 / 2}}\left\{\left(I_{2} \tilde{W}_{2}+I_{3} \tilde{W}_{3}\right) \mathbf{I}+\tilde{W}_{1} \mathbf{B}-I_{3} \tilde{W}_{2} \mathbf{B}^{-1}\right. \\
& +\left(I_{4} \tilde{W}_{9}+I_{10} \tilde{W}_{10}\right) \mathbf{a} \otimes \mathbf{a}+\left(I_{6} \tilde{W}_{9}+I_{10} \tilde{W}_{10}\right) \mathbf{b} \otimes \mathbf{b} \\
& +\frac{1}{2} I_{10}^{1 / 2} \tilde{W}_{8}(\mathbf{a} \otimes \mathbf{b}+\mathbf{b} \otimes \mathbf{a})+I_{4} \tilde{W}_{11}(\mathbf{a} \otimes \mathbf{B a}+\mathbf{B a} \otimes \mathbf{a}) \\
& \left.+I_{6} \tilde{W}_{11}(\mathbf{b} \otimes \mathbf{B b}+\mathbf{B b} \otimes \mathbf{b})\right\},
\end{aligned}
$$

where $\tilde{W}_{i}$ is the partial derivative of $\tilde{W}$ with respect to the $i$ th invariant and $\mathbf{I}$ is the identity tensor. Finally, we specialize to the case of pure stretch deformations, which are the type of experimental deformations we are proposing to study. A pure stretch deformation takes the form

$\mathbf{F}=\lambda_{1} \mathbf{E}_{1} \otimes \mathbf{E}_{1}+\lambda_{2} \mathbf{E}_{2} \otimes \mathbf{E}_{2}+\lambda_{3} \mathbf{E}_{3} \otimes \mathbf{E}_{3}$.

Hence, using the deformation gradient tensor (7) we can calculate the invariants in (5) and, using (6), can write the Cauchy stress explicitly in terms of the principle stretches as

$$
\begin{aligned}
\mathbf{T}= & \frac{2}{\lambda_{1} \lambda_{2} \lambda_{3}}\left\{\left[\tilde{W}_{2}\left(\lambda_{1}^{2} \lambda_{2}^{2}+\lambda_{1}^{2} \lambda_{3}^{2}+\lambda_{3}^{2} \lambda_{2}^{2}\right)+\tilde{W}_{3} \lambda_{1}^{2} \lambda_{2}^{2} \lambda_{3}^{2}\right]\right. \\
& \times\left(\mathbf{E}_{1} \otimes \mathbf{E}_{1}+\mathbf{E}_{2} \otimes \mathbf{E}_{2}+\mathbf{E}_{3} \otimes \mathbf{E}_{3}\right) \\
& +\tilde{W}_{1}\left(\lambda_{1}^{2} \mathbf{E}_{1} \otimes \mathbf{E}_{1}+\lambda_{2}^{2} \mathbf{E}_{2} \otimes \mathbf{E}_{2}+\lambda_{3}^{2} \mathbf{E}_{3} \otimes \mathbf{E}_{3}\right) \\
& -\left(\lambda_{1} \lambda_{2} \lambda_{3}\right)^{2} \tilde{W}_{2}\left(\lambda_{1}^{-2} \mathbf{E}_{1} \otimes \mathbf{E}_{1}+\lambda_{2}^{-2} \mathbf{E}_{2} \otimes \mathbf{E}_{2}\right. \\
& \left.+\lambda_{3}^{-2} \mathbf{E}_{3} \otimes \mathbf{E}_{3}\right)+2\left[\tilde{W}_{9}+\left(\lambda_{2}^{2} \sin ^{2} \Phi\right.\right. \\
& \left.\left.+\lambda_{3}^{2} \cos ^{2} \Phi\right) \tilde{W}_{10}\right]\left(\lambda_{2}^{2} \sin ^{2} \Phi \mathbf{E}_{2} \otimes \mathbf{E}_{2}\right. \\
& \left.+\lambda_{3}^{2} \cos ^{2} \Phi \mathbf{E}_{3} \otimes \mathbf{E}_{3}\right)+\tilde{W}_{8}\left(-\lambda_{2}^{2} \sin ^{2} \Phi \mathbf{E}_{2} \otimes \mathbf{E}_{2}\right. \\
& \left.+\lambda_{3}^{2} \cos ^{2} \Phi \mathbf{E}_{3} \otimes \mathbf{E}_{3}\right)+4 \tilde{W}_{11}\left(\lambda_{2}^{4} \sin ^{2} \Phi \mathbf{E}_{2} \otimes \mathbf{E}_{2}\right. \\
& \left.\left.+\lambda_{3}^{4} \cos ^{2} \Phi \mathbf{E}_{3} \otimes \mathbf{E}_{3}\right)\right\} .
\end{aligned}
$$

Due to our assumptions, our fiber-reinforced continuum is orthotropic with planes of reflectional symmetry which are normal to the basis vectors $\left(\mathbf{E}_{1}, \mathbf{E}_{2}, \mathbf{E}_{3}\right)$. Hence, Eq. (6) for our fiber-reinforced continuum can be shown to be equivalent to an orthotropic representation. However, an advantage of using the fiber-reinforced continuum theory is that if two annular specimens are mechanically equivalent and differ only by the collagen fiber orientations, then a single constitutive Eq. (6) can be applied to generate the differing mechanical properties by changing only the fiber angle.

\subsection{Specification of the strain energy function}

Motivated by the approach of Almeida et al. (1995) for a transversely isotropic model, we first considered the 
following $W$ as an approximation to the strain energy function for use with the fiber-reinforced theory

$$
\begin{aligned}
W= & \frac{1}{I_{3}^{n}} \beta_{0} \exp \left[\beta_{1}\left(I_{1}-3\right)+\beta_{2}\left(I_{2}-3\right)+\beta_{3}\left(I_{1}-3\right)^{2}\right. \\
& +\beta_{4}\left(I_{9}-2\right)+\beta_{5}\left(I_{9}-2\right)^{2} \\
& +\beta_{6}\left(I_{1}-3\right)\left(I_{9}-2\right)+\beta_{7}\left(I_{11}-2\right) \\
& +\beta_{8}\left(I_{10}-1\right)+\beta_{9}\left(I_{8}-\cos ^{2} 2 \Phi\right)^{2} \\
& +\beta_{10}\left(I_{1}-3\right)\left(I_{8}-\cos ^{2} 2 \Phi\right) \\
& \left.+\beta_{11}\left(I_{8}-\cos ^{2} 2 \Phi\right)\left(I_{9}-2\right)\right],
\end{aligned}
$$

where $n$ and $\beta_{i}, i=(0,1,2,3,4,5,6,7,8,9,10,11)$ are material constants. The constant $\beta_{0}$ has dimensions of stress while the remaining constants are dimensionless. By demanding that there exists a stress free reference configuration, we derived the following relationships:

$n=\beta_{1}+2 \beta_{2}, \quad \beta_{7}=-(1 / 2)\left(\beta_{4}+\beta_{8}\right)$

so that we have eleven independent material constants $\left(\beta_{0}, \beta_{1}, \beta_{2}, \beta_{3}, \beta_{4}, \beta_{5}, \beta_{6}, \beta_{8}, \beta_{9}, \beta_{10}, \beta_{11}\right)$. The motivations for choosing the form (9) for $W$ were: (1) by setting $\mathbf{b}_{0}=\mathbf{0}$ to recover a one-fiber family theory (i.e., transverse isotropy), it reduces to that used by Almeida et al. (1995); (2) extra terms involving the invariants were added so that it reduces to the most general quadratic expression suggested by Spencer (1984) for use in a linear theory; and (3) these extra terms were included in the single exponential as proposed by Almeida et al. (1995).

In a preliminary investigation we experienced difficulty using this formulation to specify the material parameters so as to obtain a reasonable fit to the stress vs. stretch data from four independent experiments. Hence, we chose another approximation to the strain energy function that places the dependence of the invariants in separate exponentials, instead of the same exponential as in (9). This second approximation to the strain energy function was

$$
\begin{aligned}
W= & \alpha_{0}\left\{\exp \left[\alpha_{1}\left(I_{1}-3\right)\right]+\exp \left[\alpha_{2}\left(I_{2}-3\right)\right]\right. \\
& +\exp \left[\alpha_{3}\left(I_{3}-1\right)\right]+\exp \left[\alpha_{4}\left(I_{9}-2\right)\right] \\
& +\exp \left[\alpha_{5}\left(I_{8}-\cos ^{2} 2 \Phi\right)^{2}\right]+\exp \left[\alpha_{6}\left(I_{10}-1\right)\right] \\
& +\exp \left[\alpha_{7}\left(I_{11}-2\right)\right]+\exp \left[\alpha_{8}\left(I_{1}-3\right)\left(I_{8}-\cos ^{2} 2 \Phi\right)\right] \\
& +\exp \left[\alpha_{9}\left(I_{1}-3\right)\left(I_{9}-2\right)\right] \\
& \left.+\exp \left[\alpha_{10}\left(I_{8}-\cos ^{2} 2 \Phi\right)\left(I_{9}-2\right)\right]\right\}
\end{aligned}
$$

where $\alpha_{i}, i=(0,1,2,3,4,5,6,7,8,9,10)$ are material constants. The constant $\alpha_{0}$ has dimensions of stress while the remaining constants are dimensionless. By demanding a stress free reference configuration, we derived

$\alpha_{3}=-\left(\alpha_{1}+2 \alpha_{2}\right), \quad \alpha_{7}=-(1 / 2)\left(\alpha_{4}+\alpha_{6}\right)$, so that we have nine independent material constants $\left(\alpha_{0}, \alpha_{1}, \alpha_{2}, \alpha_{4}, \alpha_{5}, \alpha_{6}, \alpha_{8}, \alpha_{9}, \alpha_{10}\right)$ corresponding to Eq. (11). The motivations for choosing the form in Eq. (11) for $W$ were: (1) it contains the necessary terms involving the invariants so that it reduces to the most general quadratic expression suggested by Spencer (1984) for use in a linear theory, resulting in 9 independent material constants for a linear orthotropic material; and (2) the dependence on the various invariants were expressed in separate exponentials which we believe allows for more flexibility in simultaneously matching data from several experimental protocols. We chose an exponential form instead of a polynomial for both strain energy functions as Fung et al. (1997) have shown that the exponential form results in smaller coefficients of variation in material constants determined from experimental data of biological tissues.

\subsection{Experimental data}

The experimental data to which we applied the strain energy functions (9) and (11) included: (A) confined compression, radial stress vs. radial stretch, nondegenerate anterior outer (Iatridis et al., 1998); (B) confined compression, axial stress vs. axial stretch, nondegenerate anterior outer (Iatridis et al., 1998); (C) simple tension, radial stress vs. radial stretch, nondegenerate anterior middle (Fujita et al., 1998); (D) simple tension, circumferential stress vs. circumferential stretch, nondegenerate anterior outer and inner (Ebara et al., 1996) (Fig. 2). We assumed that this experimental data represents the equilibrium elastic (i.e., drained) response of the tissue in the context

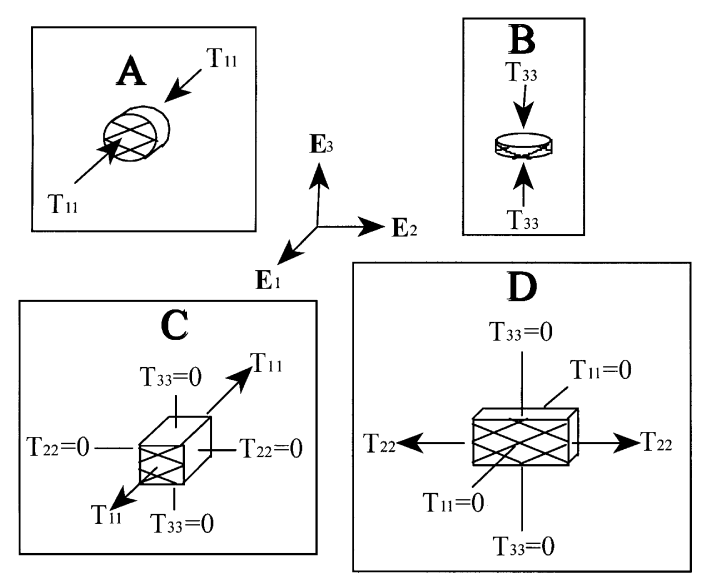

Fig. 2. Schematic reqresentation of the applied stresses for the four experimental protocols: A - radial confined compression; B - axial confined compression; C - radial tension; D - circumferential tension. The basis vectors $\left(\mathbf{E}_{1}, \mathbf{E}_{2}, \mathbf{E}_{3}\right)$ are aligned in the local radial, circumferential, and axial directions, respectively. The traction-free boundary conditions in experiment $\mathrm{C}$ are satisfied by setting the circumferential stress $T_{22}$ and the axial stress $T_{33}$ to zero. The tractionfree boundary conditions in experiment $\mathrm{D}$ are satisfied by setting the radial stress $T_{11}$ and the axial stress $T_{33}$ to zero. 
of the nonlinear biphasic theory proposed by Holmes and Mow (1990), and that the equilibrium response represents a homogeneous stress state. For (A) and (B), we set the principal stretches in the two directions that were normal to the loading direction equal to unity. For (C) and (D), we had to make assumptions concerning the principal stretches that were not directly measured. In using the data from (C), we estimated $\lambda_{2}=$ $\lambda_{3}=1.25-0.25 \lambda_{1}$, where the stretch in the radial direction, $\lambda_{1}$, was measured. These relations were derived by using unpublished data gathered from our laboratory in which radial tension experiments were conducted while measuring the tissue stretches (i.e., contraction) on the free surfaces of the tissue. For (D), we estimated $\lambda_{1}=1 ; \lambda_{3}=2-\lambda_{2}$, where the stretch in the circumferential direction, $\lambda_{2}$, was measured. The relation for $\lambda_{3}$ was derived using a Poisson's ratio of 1.0 as Acaroglu et al. (1995) reported a Poisson's ratio for this stretch of $1.0-1.25$ for normal, anterior outer annulus in circumferential tensile tests. We assumed that the contraction in the radial direction $\left(\lambda_{1}\right)$ would be substantially less than the contraction in the fiber direction $\left(\lambda_{3}\right)$ and chose a constant value for $\lambda_{1}$ of 1.0. For (C) and (D), we converted (8) to the first Piola-Kirchhoff stress $\mathbf{P}$, since experimental stresses were calculated by dividing by the original cross sectional areas. For all experiments, we assumed a uniform fiber angle of $\Phi=60^{\circ}$ from the axial direction.

We conducted simultaneous regressions to the four experimental data sets (A)-(D) with and without invoking the traction-free boundary conditions for (C) and (D). In general, when solving the boundary value problem for the radial tension test $(\mathrm{C})$ one must insure that the free surface stresses in the circumferential and axial directions, $T_{22}$ and $T_{33}$, equal zero (Fig. 2). Hence, when invoking the traction-free boundary conditions for (C), we derived equations for the axial and circumferential stresses, $T_{22}$ and $T_{33}$, in terms of the radial stretch, $\lambda_{1}$, and set these equations equal to zero and included them in the set of equations used in the simultaneous regression. Similarly, when invoking the traction-free boundary conditions for (D), we derived equations for the axial and radial stresses, $T_{33}$ and $T_{11}$, in terms of the circumferential stretch, $\lambda_{2}$, which were set equal to zero (Fig. 2). Initially, regressions were performed without invoking the traction-free boundary conditions as this may be a better relative evaluation of the two proposed strain energy functions as considerable uncertainty in the free surface stretches, which were not directly measured in the tensile experiments, may introduce large errors in the predictions of the free surface stresses.

The derivatives of $W$ with respect to each of the invariants were calculated from Eqs. (9) and (11) for our two choices of the strain energy function, and were introduced into Eq. (8) to derive the stress vs. stretch relationships using Mathematica (V. 2.2.1, Wolfram Research, Cham- paign, IL). The resulting stress vs. stretch equations predicted by the theory and the specific choices of the strain energy function are too long to present here.

\subsection{Statistical analysis}

To prescribe the material coefficients imbedded in our formulation, we conducted a simultaneous nonlinear regression to the mean response of the stress versus stretch data from the four experiments using a Levenberg-Marquardt nonlinear fitting algorithm (Mathematica). This was accomplished by first creating a composite function, $T^{\prime}$, that was the sum of the measured stress vs. stretch equations from the four experimental protocols

$T^{\prime}=\sum_{j=1}^{4} \sum_{i=1}^{4} T_{i} \delta_{i j}$

where $\delta_{i j}$ is the Kroneker delta function and $T_{i}$ is the stress versus stretch solution for the $i$ th experiment. This composite function was then fit to the four data sets to determine the material coefficients. In this manner, the goodness of fit statistic $\left(\chi^{2}\right)$ calculated for $T^{\prime}$ was a measure of how well the underlying constitutive formulation simultaneously described the four experimental data sets. We first performed the simultaneous, nonlinear regression analysis to the mean experimental response corresponding to the stress versus stretch data which were directly measured in the loading direction of experiments (A)-(D). We repeated the simultaneous, nonlinear regression using the four equations mentioned above plus four additional equations corresponding to the traction-free boundary conditions for experiments (C) and (D) to determine a new set of the material constants. We rejected any solutions for the material constants for either strain energy function that predicted non-monotonic behavior for any of the measured stress-stretch relationships in the range of experimentally reported stretches.

\section{Results}

The strain energy given by (11) resulted in the lower $\chi^{2}$ value in the simultaneous regression to the four directly measured stress-stretch relations. Using the strain energy given by Eq. (9), the best-fit values of $\left(\beta_{0}, \beta_{1}, \beta_{2}, \beta_{3}, \beta_{4}, \beta_{5}, \beta_{6}, \beta_{8}, \beta_{9}, \beta_{10}, \beta_{11}\right) \quad$ were $(0.204$, $0.751,0.075,-0.291,0.647,3.868,-0.399,1.364$, $-0.084,2.004,1.045)$. With these values, the predicted stress versus stretch composite function (13) was correlated to the experimental data with a $\chi^{2}$ value of 0.35 (Fig. 3). Using the strain energy given by Eq. (11), the best-fit values of $\left(\alpha_{0}, \alpha_{1}, \alpha_{2}, \alpha_{4}, \alpha_{5}, \alpha_{6}, \alpha_{8}, \alpha_{9}, \alpha_{10}\right)$ were $(0.001,-0.737,-3.289,-6.496,0.4091,17.713$, 
A

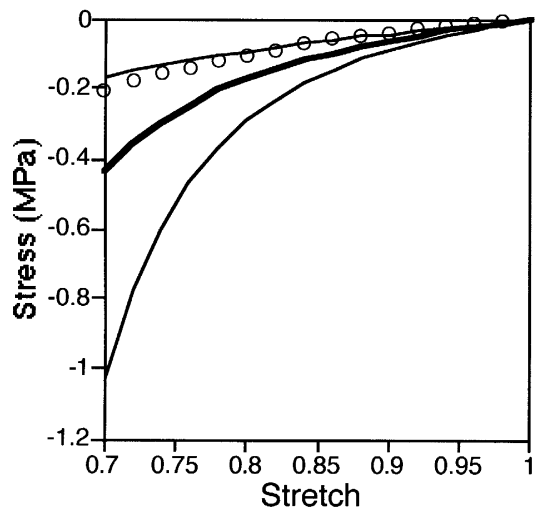

$\mathrm{C}$

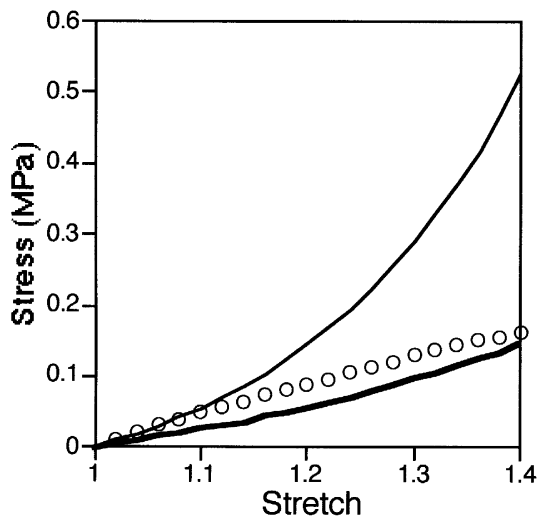

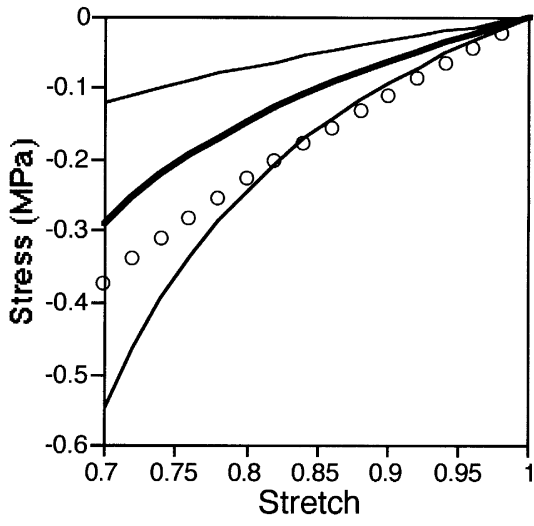

$\mathrm{D}$

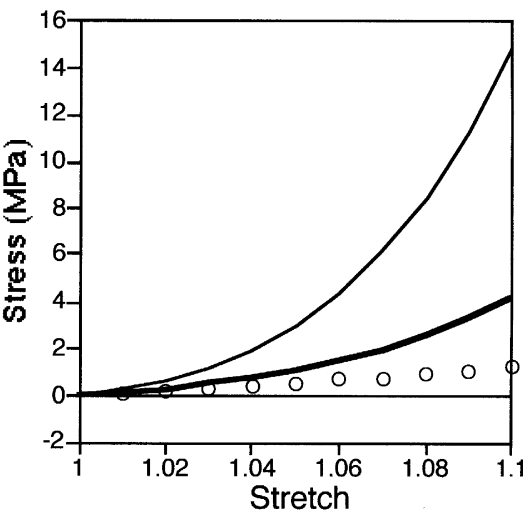

Fig. 3. Simultaneous curve-fit results using the strain energy of Eq. (9) to match the four equations representing the measured stress-stretch data. The material constants $\left(\beta_{0}, \beta_{1}, \beta_{2}, \beta_{3}, \beta_{4}, \beta_{5}, \beta_{6}, \beta_{8}, \beta_{9}, \beta_{10}, \beta_{11}\right)$ equal to $(0.204,0.751,0.075,-0.291,0.647,3.868,-0.399,1.364,-0.084,2.004,1.045)$ resulted in a $\chi^{2}$ value of 0.35 . A $=$ radial confined compression (Iatridis et al., 1998); B = axial confined compression (Iatridis et al., 1998); $\mathrm{C}=$ radial tension (Fujita et al., 1997); D = circumferential tension (Ebara et al., 1996). Solid curves = mean experimental response \pm 1 standard deviation; open circles $=$ theoretical prediction of measured stress-stretch response.

$0.683,-2.615,0.262)$. With these values, the predicted stress versus stretch composite function (13) was correlated to the experimental data with a $\chi^{2}$ value of 0.08 (Fig. 4).

The strain energy given by Eq. (11) resulted in the lower $\chi^{2}$ value in the simultaneous regression to the four directly measured stress-stretch relations plus the four additional equations corresponding to the traction-free boundary conditions for experiments (C) and (D). Using the strain energy given by Eq. (9), the best-fit values of $\left(\beta_{0}, \beta_{1}, \beta_{2}, \beta_{3}, \beta_{4}, \beta_{5}, \beta_{6}, \beta_{8}, \beta_{9}, \beta_{10}, \beta_{11}\right)$ were $(-0.0004$, $7.399,-0.869,-3.702,-4.489,10.163,12.112,871.94$, $-352.91,-71.83,162.42)$. With these values, the predicted stress versus stretch composite function (13) was correlated to the experimental data with a $\chi^{2}$ value of 1.15. Using the strain energy given by Eq. (11), the best-fit values of $\left(\alpha_{0}, \alpha_{1}, \alpha_{2}, \alpha_{4}, \alpha_{5}, \alpha_{6}, \alpha_{8}, \alpha_{9}, \alpha_{10}\right)$ were (0.001, $-1.716,-3.338, \quad-4.574,0.369, \quad 12.955, \quad 0.600$, $-2.911,0.212)$. With these values, the predicted stress versus stretch composite function (13) was correlated to the experimental data with a $\chi^{2}$ value of 0.51 (Fig. 5).

\section{Discussion}

We have developed a nonlinear, orthotropic constitutive theory for the annulus that matches current experimental data representing the broad range of stresses anticipated in vivo. We utilized a conceptually novel technique of simultaneous regression to multiple data sets in order to determine the material constants. Using a theory for an isotropic continuum reinforced with two families of fibers, we were able to specify a formulation whose predictions simultaneously fell within one standard deviation of the mean response of the annulus when measured in four different experimental deformations. Good approximation to the data was achieved by using a strain energy function (11) which expresses its dependence on the invariants in separate, multiple exponentials instead of combining all of the invariant terms in a single exponential. This finding is especially noteworthy as the single exponential form of the strain energy function (11) used two less material constants than that incorporating multiple exponentials (9). 
A

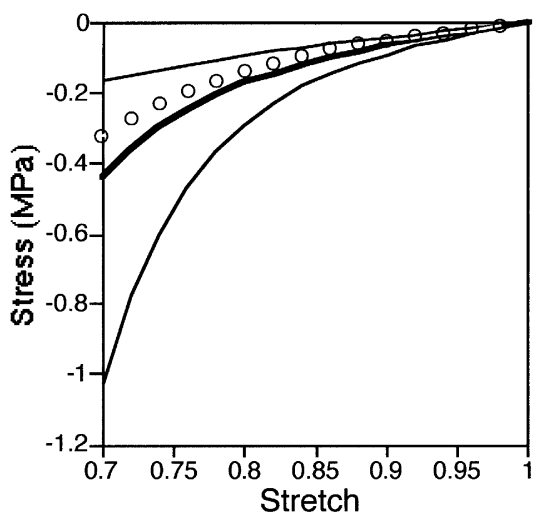

C

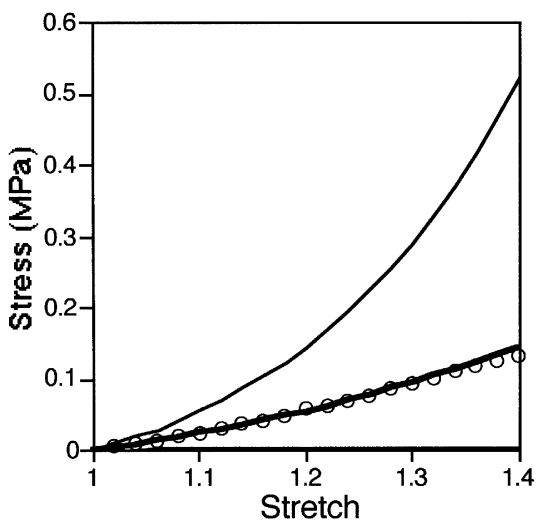

B

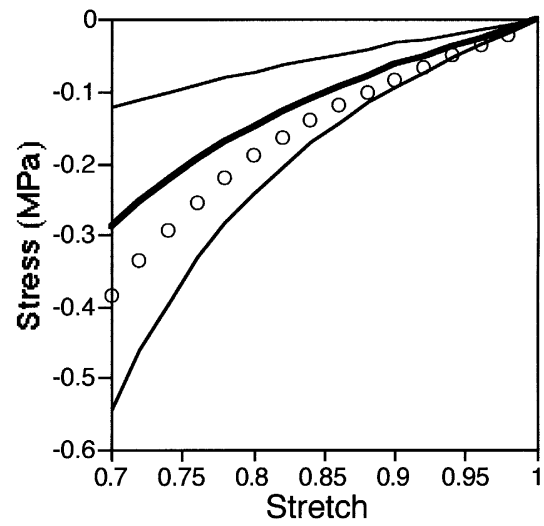

D

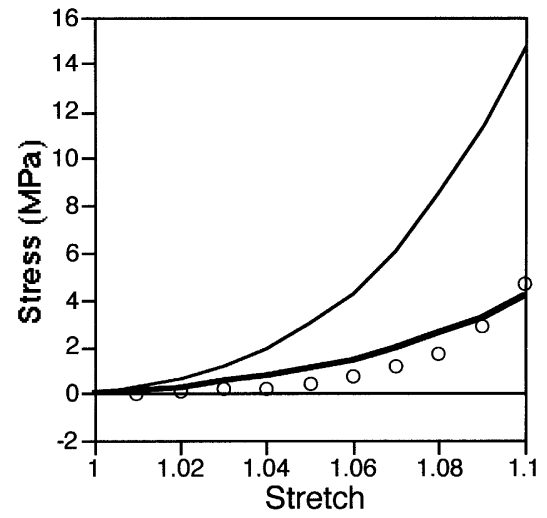

Fig. 4. Simultaneous curve-fit results using the strain energy of Eq. (11) to match the four equations representing the measured stress-stretch data. The material constants $\left(\alpha_{0}, \alpha_{1}, \alpha_{2}, \alpha_{4}, \alpha_{5}, \alpha_{6}, \alpha_{8}, \alpha_{9}, \alpha_{10}\right)$ equal to $(0.001,-0.737,-3.289,-6.496,0.409,17.713,0.683,-2.615,0.262)$ resulted in a $\chi^{2}$ value of 0.08 . A = radial confined compression (Iatridis et al., 1998); B = axial confined compression (Iatridis et al., 1998); $\mathrm{C}=$ radial tension (Fujita et al., 1997); D = circumferential tension (Ebara et al., 1996). Solid curves = mean experimental response \pm 1 standard deviation; open circles $=$ theoretical prediction of measured stress-stretch response.

The theory proposed in the present paper is similar to that used by Wu and Yao (1976), who also investigated the mechanical properties of the annulus using Spencer's theoretical approach. They developed the theory for a material with two families of extensible fibers and used a polynomial form of the strain energy function. The primary difference between the work of the present paper and that of $\mathrm{Wu}$ and Yao (1976) is that the current model is compressible, which is compatible with current experimental and theoretical observations (Iatridis et al., 1998; Holmes and Mow, 1990). Also, our proposed strain energy function is a combination of exponential functions instead of a polynomial and was fit to experimental data from four distinct protocols. Our strain energy function is consistent with that of Fung et al. (1979), who suggested that the exponential form has more flexibility in matching experimental data and thus may be used successfully with fewer material coefficients. The present study also demonstrates the need to simultaneously consider the annular behavior under multiple deformations. In particular, when fitting the model independently to each of the stress-stretch curves in a preliminary study (Klisch and Lotz, 1998b), we obtained significantly different material constants for each experimental data set. That is, a model based on one type of experiment could not predict the response of the same tissue under different boundary conditions. These results demonstrate that significant errors may be encountered when attempting to extrapolate the theoretical predictions beyond those explicitly included in the development of the material law. Also, there are fewer equations than unknown material constants in both regressions that were performed in the present paper and which indicates that the solutions obtained are not unique. Thus, equations gained from modeling additional deformations would be useful in identifying unique solutions for the material constants of the strain energy function.

We have not invoked an a priori constitutive inequality to obtain restrictions on the material constants which appear in the proposed strain energy functions. A few constitutive inequalities which have been proposed in the past include the Baker-Ericksen inequalities, convexity, 
A

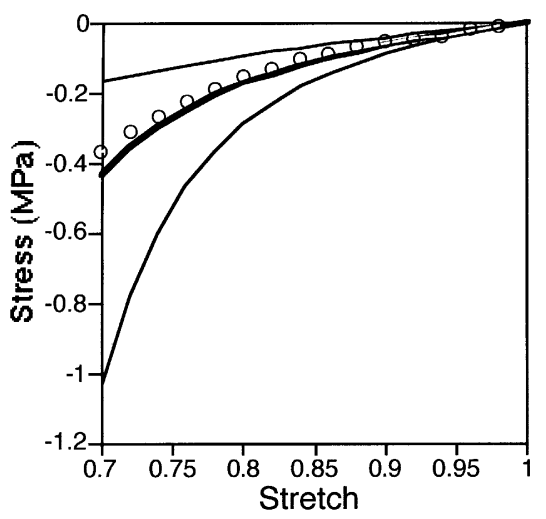

C

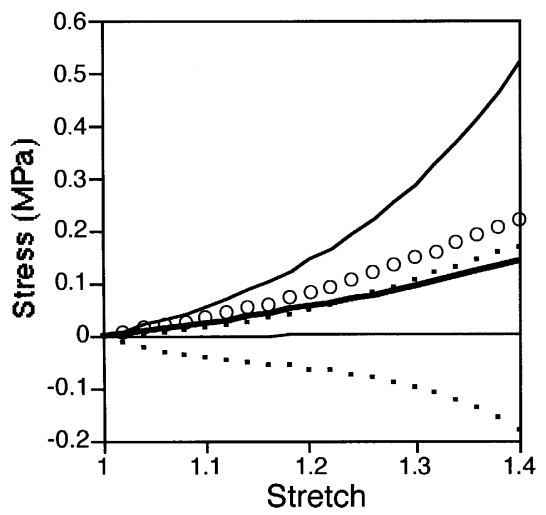

B

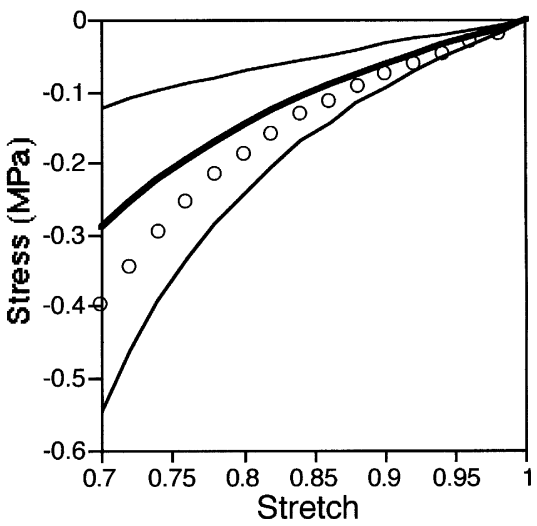

$\mathrm{D}$

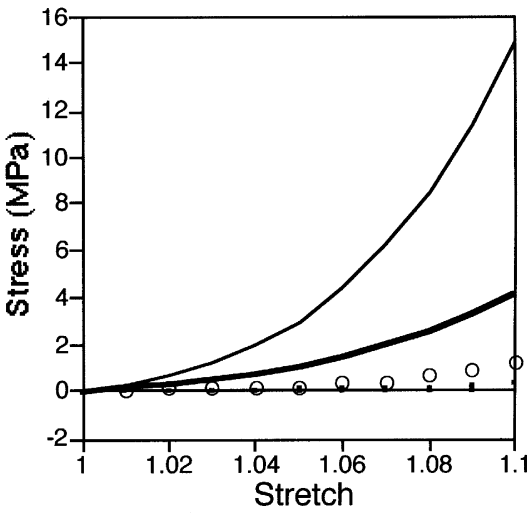

Fig. 5. Simultaneous curve-fit results using the strain energy of Eq. (11) to match the four equations representing the measured stress-stretch data and the additional four equations representing the traction-free boundary conditions for experiments $\mathrm{C}$ and $\mathrm{D}$. The material constants $\left(\alpha_{0}, \alpha_{1}, \alpha_{2}, \alpha_{4}, \alpha_{5}, \alpha_{6}, \alpha_{8}, \alpha_{9}, \alpha_{10}\right)$ equal to $(0.001,-1.716,-3.338,-4.574,0.369,12.955,0.600,-2.911,0.212)$ resulted in a $\chi^{2}$ value of 0.51 . $\mathrm{A}=$ radial confined compression (Iatridis et al., 1998); B = axial confined compression (Iatridis et al., 1998); C = radial tension (Fujita et al., 1997); $\mathrm{D}=$ circumferential tension (Ebara et al., 1996). Solid curves $=$ mean experimental response \pm 1 standard deviation; open circles $=$ theoretical prediction of measured stress-stretch response; filled squares $=$ theoretical prediction of 'traction-free' stress-stretch response for experiments $\mathrm{C}$ and D.

the generalized Coleman-Noll condition (Truesdell and Noll, 1965), and the requirement that the strain energy becomes unbounded as the Jacobian of the deformation gradient approaches zero (Holmes and Mow, 1990; Kwan et al., 1990). Apart from the last condition mentioned above, it is not clear that these inequalities are appropriate for anisotropic continua, as the BakerEricksen inequalities are only postulated for isotropic continua and both convexity and the generalized Coleman-Noll condition are regarded as too restrictive (Marsden and Hughes, 1983). Nevertheless, we investigated whether the stress vs. stretch response predicted when using our proposed strain energy function (11) remained convex over a physiologic range of elastic stretches. The predicted stress-stretch relations remained convex to stretches of 0.3 in the confined compression protocols, which would be the maximum allowed stretch based on an initial solid content of 0.3 (Best et al., 1994) and the biphasic constraint of intrinsic incompressibility (Holmes and Mow, 1990). In addition, convexity was predicted in tensile tests beyond the reported failure stretches of 1.8 for a radial tension protocol (Fujita et al., 1997) and 1.15 for a circumferential tension protocol (Ebara et al., 1996). Since strict convexity is a sufficient condition for incremental stability and uniqueness (Ogden, 1997), we regard the proposed strain energy function (11), along with the reported material constants, as being physically plausible for the range of deformations studied.

The inclusion of the traction-free boundary conditions in the simultaneous regression resulted in a poorer overall fit to the experimental data. The likely reason for this is that we needed to assume the principal stretches in the off-axis directions for the tension experiments (C and D) since these were neither measured nor reported in the literature. For example, when studying the radial tension response, the traction-free stress components lie in the axial and circumferential directions of the tissue which are much stiffer than the radial direction of applied 
loading due to the collagen fiber orientation. As a result, small errors in the estimates of the axial and circumferential stretches will lead to large relative errors in the predicted stresses in these directions as compared to the measured stresses in the radial direction. This is the primary reason we chose to also conduct regressions without invoking the traction-free boundary conditions. Other sources of error include the lack of explicit data regarding the initial fiber orientations and the assumption that the fiber angle was constant throughout the specimens.

Another limitation of the present study is the large variation in the available experimental data for the annulus. As a result, some differences observed for the stress vs. stretch responses between the four experimental protocols may be attributed to specimen-specific variability as opposed to tissue anisotropy. While this may be true, the stress required to produce a stretch of 1.1 for the circumferential tension experiment was two orders of magnitude higher than that for the radial tension experiment, indicating a strong anisotropic response of the annulus. Also, in the future the influence of specimenspecific variability may be reduced by including additional parameters (donor, disc level, and disc region) into the statistical regression. Despite these limitations, the work of the present paper is unique in that it is the first time that a nonlinear anisotropic continuum theory for the annulus fibrosus has been simultaneously applied to multiple experimental protocols. The success of this approach is encouraging and demonstrates both the need and feasibility of combining data from multiple experiments to define a constitutive theory for annular tissue. In order to improve the predictive capability of the fiberreinforced theory, we plan on conducting additional experimental and theoretical studies including biaxial tension experiments (Bass and Lotz, 1999) and including shear deformations in the regression (Wagner et al., 1999; Fujita et al., 1996).

Another approach to modeling the nonlinear, anisotropic behavior of the annulus may be to develop a continuum mixture model by representing the ground substance and collagen fibers as individual solids (Atkin and Craine, 1976) or by employing homogenization techniques (Suquet, 1985). These approaches may provide a better prediction of the stresses acting on the ground substance, the individual collagen fibers, and the cells, and should ultimately be sought to explore the relationship between applied stresses and the cellular biosynthetic response. However, such nonlinear theories are substantially more complex than the anisotropic model proposed in the present paper. In particular, they pose the difficulty of experimentally determining the material properties of the matrix constituents, specifying the applied experimental tractions to each of the constituents (i.e., the ground substance and the collagen fibers), and require additional constitutive equations related to the microstructure (i.e., matrix-fiber interactions). The theory which we have proposed appears to be sufficient for realizing our goal of developing an anisotropic continuum model of the annulus capable of simultaneously predicting the macroscopic stresses measured in multiple experimental protocols. Yet, the question of how precise tissue stress predictions need to be, and consequently the appropriate method to be used, can only be answered within the context of specific research questions raised in studies where these theoretical techniques are applied. Nonetheless, our results demonstrate that regardless of the theoretical approach utilized, validation with data from a variety of experimental deformations is crucial for accurately modeling the behavior of tissues with strong anisotropy such as the annulus fibrosus.

Another advantage of the fiber-reinforced continuum theory over standard orthotropy is that once a constitutive equation is obtained for a specimen, then the constitutive equation for another specimen which only differs by the orientation of the collagen fibers can be obtained by adjusting a single parameter, the fiber angle. Since it has been shown that the fiber angle from the axial direction varies from $\pm 60^{\circ}$ at the periphery to $\pm 45^{\circ}$ at the innermost layers (Cassidy et al., 1989), the fiber-reinforced theory has the potential for separating the effects of heterogeneous collagen fiber orientation from other microstructural parameters (e.g., collagen fiber density and proteoglycan content). We expect that the material constants used in the present theory would depend strongly on collagen content, proteoglycan content, and degeneration grade of the disc. For these reasons, we believe that the proposed fiber-reinforced continuum theory is a logical first step in characterizing the strongly anisotropic behavior of the annulus, and future studies should include data from other deformations (such as shear), the precise measurement of all three stretches in tensile experiments, and specimen-specific regressions.

In summary, we have developed a fiber-reinforced continuum model of the annulus fibrosus and simultaneously applied the theory to the mean response measured in four distinct experimental protocols undergoing large deformations. In doing so we have identified a new exponential form for the annular strain energy function that is dependent on nine material coefficients. We anticipate that the utilization of the proposed constitutive law will result in more accurate predictions of the anisotropic stresses and strains developed within the annulus in vivo in both health and disease and ultimately will lead to a better understanding of the mechanisms of degenerative disc disease.

\section{Acknowledgements}

This research was sponsored by funding from UCSF REAC and the National Institutes of Health (AR44179). 


\section{References}

Acaroglu, E.R., Iatridis, J.C., Setton, L.A., Foster, R.J., Mow, V.C., Weidenbaum, M., 1995. Degeneration and aging affect the tensile behavior of human lumbar anulus fibrosus. Spine 20 (24), 2690-2701.

Almeida, E.S., Spilker, R.L., Holmes, M.H., 1995. A transversely isotropic constitutive law for the solid matrix of articular cartilage. Advances in Bioengineering ASME 29, 161-162.

Atkin, R.J., Craine, R.E., 1976. Continuum theories of mixtures: applications. 153-207.

Bass, E.C., Lotz, J.C., 1999. Biaxial behavior of the annulus fibrosus in tension. Transactions of the 45th Annual Meeting, Orthopaedic Research Society, 1035.

Brickley-Parsons, D., Glimcher, J., 1984. Is the chemistry of collagen in intervertebral discs an expression of Wolff's Law? A study of the human lumbar spine. Spine 9 (12), 148-163.

Broberg, K.B., 1983. On the mechanical behavior of intervertebral discs. Spine 8 (2), 151-165.

Cassidy, J.J., Hiltner, A., Baer, E., 1989. Hierarchical structure of the intervertebral disc. Connective Tissue Research 23 (1), $75-88$.

Deyo, R.A., Cherkin, D., Conrad, D., Volinn, E., 1991. Cost, controversy, crisis: low back pain and the health of the public. Annual Review of Public Health 12, 141-156.

Duncan, N.A., Lotz, J.C., 1998. Experimental validation of a porohyperelastic finite element model of the annulus fibrosus. In: Computer Methods in Biomechanics and Biomedical Engineering - 2. Gordon and Breach, London, pp. 527-534.

Ebara, S., Iatridis, J.C., Setton, L.A., Foster, R.J., Mow, V.C., Weidenbaum, M., 1996. Tensile properties of nondegenerate human lumbar anulus fibrosus. Spine 21 (4), 452-461.

Elliott, D.M., LeRoux, M.A., Laursen, T.A., Setton, L.A., 1997. Formulation of a continuum anistotropic model for the annulus fibrosus in tension. Advances in Bioengineering, ASME 36, 165-166.

Fujita, Y., Duncan, N.A., Lotz, J.C., 1996. Anisotropic shear behavior of the annulus fibrosus: effect of harvest site and tissue prestrain. Transactions of the 42nd Annual Meeting, Orthopaedic Research Society, p. 271.

Fujita, Y., Duncan, N.A., Lotz, J.C., 1997. Radial tensile properties of the lumbar annulus fibrosus are site and degeneration dependent. Journal of Orthopaedic Research 15 (6), 814-819.

Fung, Y.C., Fronek, K., Patitucci, P., 1979. Pseudoelasticity of arteries and the choice of its mathematical expression. American Journal of Physiology 237 (5), H620-H631.

Galante, J.O., 1967. Tensile properties of the human lumbar annulus fibrosus. Acta Orthopaedica Scandanavia (Suppl. 100), S1-S91.

Holmes, M.H., Mow, V.C., 1990. The nonlinear characteristics of soft gels and hydrated connective tissues in ultrafiltration. Journal of Biomechamics 23, 1145-1156.

Iatridis, J.C., Setton, L.A., Foster, R.J., Rawlins, B.A., Weidenbaum, M., Mow, V.C., 1998. Degeneration affects the anisotropic and nonlinear behaviors of human anulus fibrosus in compression. Journal of Biomechamics 31, 535-544.
Klisch, S.M., Lotz, J.C., 1998a. A fiber-reinforced continuum theory and constitutive equation for the annulus fibrosus. Transactions of the 44th Annual Meeting, Orthopaedic Research Society, p. 1056.

Klisch, S.M., Lotz, J.C., 1998b. Application of a fiber-reinforced continuum theory to multiple deformations of annulus fibrosus. Advances in Bioengineering, ASME 39, 237.

Kwan, M.K., Lai, M.W., Mow, V.C., 1990. A finite deformation theory for cartilage and other soft hydrated connective tissues - I. Equilibrium results. Journal of Biomechamics 23, 145-155.

Lipson, S.J., 1988. Metaplastic proliferative fibrocartilage as an alternative concept to herniated intervertebral disc. Spine 13 (9), $1055-1060$.

Lotz, J.C., Colliou, O.K., Chin, J.R., Duncan, N.A., Liebenberg, E., 1998. Compression-induced degeneration of the intervertebral disc: an in vitro mouse model and finite element study. Spine 23 (2), 2493-2506.

Marchand, F., Ahmed, A.M., 1990. Investigation of the laminate structure of lumbar disc anulus fibrosus. Spine 15 (5), 402-410.

Marsden, J.E., Hughes, J.R., 1983. Mathematical Foundations of Elasticity. Dover, New York.

McNally, D.S., Arridge, R.G.C., 1995. An analytical model of intervertebral disc mechanics. Journal of Biomechanics 28, 53-68.

Ogden, R.W., 1997. Non-Linear Elastic Deformations. Dover, New York.

Shirazi-Adl, A., 1989. On the fibre composite material models of disc annulus - comparison of predicted stresses. Journal of Biomechanics 22, 357-365.

Shirazi-Adl, A., Ahmed, A.M., Shrivastava, S.C., 1986. A finite element study of a lumbar motion segment subjected to pure sagittal plane moments. Journal of Biomechanics 19, 331-350.

Spencer, A.J.M., 1984. Continuum Theory of the Mechanics of FibreReinforced Composites. Springer, New York.

Suquet, P.M., 1985. Approach by homogenization of some linear and nonlinear problems in solid mechanics. In: Boehler J.P. (Ed.), Plastic Behavior of Anisotropic Solids, Proceedings of the CNRS International Colloquium, p. 319.

Truesdell, C., Noll, W., 1965. The non-linear field theories of mechanics. In: Flügge, S. (Ed.), Handbuch der Physik, Vol. III/3. Springer, Berlin.

Tsuji, H., Hirano, N., Ohshima, H., Ishihara, H., Terahata, N., Motoe, T., 1993. Structural variation of the anterior and posterior anulus fibrosus in the development of human lumbar intervertebral disc. Spine 18 (2), 204-221.

Vernon-Roberts, B., Pirie, C., 1977. Degenerative changes in the intervertebral discs of the lumbar spine and their sequelae. Rheumatism and Rehabilitation 16, 13-21.

Wagner, D.R., Klisch, S.M., Lotz, J.C., 1999. A constitutive formulation which simultaneously predicts the response of the annulus fibrosus to seven distinct deformations. Proceedings of the 1999 Bioengineering Conference, ASME, BED-Vol. 42, 363.

Wu, H., Yao, R., 1976. Mechanical behavior of the human annulus fibrosus. Journal of Biomechanics 9, 1-7.

Yamada, H., 1970. In: Evans, F.G. (Ed.), Strength of Biological Materials. Williams and Wilkins, Baltimore. 